Revista Destaques Acadêmicos, Lajeado, v. 10, n. 4, 2018. ISSN 2176-3070

DOI: http://dx.doi.org/10.22410/issn.2176-3070.v10i4a2018.1711

http://www.univates.br/revistas

\title{
BISCOITOS TIPO COOKIES DESENVOLVIDOS A PARTIR DE FARINHA DE CASCA DE LARANJA
}

\author{
Michele Dutra Rosolen ${ }^{1}$, Laís Bresciani² ${ }^{2}$ Caroline Luísa Sprandel ${ }^{2}$, \\ Maíra Spader², Ânderson Luiz Klein², João Otávio Mezacasa Wollmuth ${ }^{2}$
}

Resumo: Esse estudo teve como objetivo desenvolver biscoitos tipo cookies a partir da utilização de farinha de casca de laranja como substituição parcial da farinha de trigo. Para a preparação dos biscoitos, a farinha de trigo foi substituída por 10, 20 e 30\% de farinha de casca de laranja. Os biscoitos foram avaliados quanto ao teor de umidade, teor de cinza, atividade de água, proteínas, lipídios, carboidratos, teor de minerais $(\mathrm{Na}, \mathrm{K}$ e Ca) e análise sensorial. Os biscoitos tipo cookies analisados estão de acordo com a legislação em relação a composição centesimal e apresentaram concentrações elevadas de cálcio e potássio, o que enriquece nutricionalmente o produto. Conclui-se que o uso da casca de laranja na fabricação de biscoitos pode ser uma alternativa para o aproveitamento desse resíduo, além de apresentar um elevado potencial nutricional.

Palavras-chave: Biscoitos. Cookies. Farinha de laranja.

\section{Introdução}

O Brasil se destaca como o maior produtor mundial de laranjas (FAO, 2017), sendo responsável por $50 \%$ da produção mundial de suco, alcançando dessa forma $85 \%$ da participação no mercado mundial (AGRINUAL, 2016).Os resíduos gerados nos processos de cítricos são importante fonte de flavonoides naturais e contém uma expressiva concentração de compostos fenólicos. De acordo com Li, Smith e Hossain (2006) as cascas de uvas são ricas em compostos fenólicos e apresentam a maior capacidade antioxidante, seguido pelas cascas de limão, tangerina e laranja.

A casca, polpa e as sementes das frutas cítricas contém antioxidantes naturais que são substâncias constituídas por vitaminas, minerais, pigmentos naturais e enzimas que são capazes de impedir o efeito de radicais livres no

1 Graduação em Nutrição. Doutoranda em Ciência e Tecnologia de Alimentos, Universidade Federal de Pelotas - UFPel.

2 Graduando em Química Industrial , Universidade do Vale do Taquari - UNIVATES. 
organismo (GUIMARÃES et al., 2010). Além disso, a casca de frutas cítricas contém compostos fenólicos que ajudam a reduzir o mau colesterol, a prevenir o câncer, doenças cardiovasculares e inflamações (MATOS, 2007).

As frutas cítricas são compostas por flavedo (parte externa), albedo (parte interna branca), membrana e vesículas (CAMARGO et al., 2007). A casca e o albedo da laranja são uma boa fonte nutricional, ricas em vitamina C, proteínas e fibras, além de apresentar elevado potencial de hidratação, podendo esses resíduos serem utilizados como complemento alimentar (CLEMENTE et al., 2012). O aproveitamento de resíduos de frutas cítricas para a produção de alguns alimentos ricos em fibras, minerais, antioxidantes e compostos fenólicos pode ser uma alternativa saudável, além de uma opção viavelmente tecnológica e econômica (ISHIMOTO et al., 2007).

Deitos et al. (2014) avaliaram o resíduo da casca da laranja na obtenção de pectina e óleo essencial e concluíram que o aproveitamento desse resíduo é viável para a obtenção dos coprodutos pectina e óleo essencial, obtendo um rendimento de $23 \%$ de pectina aproveitando o resíduo gerado na extração do óleo essencial e 2,07\% na extração de cascas de laranja expelidas secas e moídas e um rendimento de $2,5 \%$ na extração de óleo essencial da casca triturada.

Mesmo que não constituam um alimento básico como o pão, os biscoitos possuem diversas variedades e sabores e por isso são muito aceitos e consumidos. Além disso, os biscoitos são de grande durabilidade o que permite a produção em maior escala e distribuição (BRUNO e CAMARGO, 1995; CHEVALLIER et al., 2000; GUTKOSKI; NODARI; JACOBSEN NETO, 2003). O Brasil é o $2^{\circ}$ maior produtor de biscoitos do mundo e produziu, em 2016, 1.336,3 milhões de toneladas (ABIMAPI, 2017) .

Segundo a Resolução - CNNPA n ${ }^{\circ}$ 12, de 1978 da Anvisa, biscoito ou bolacha é o produto obtido pelo amassamento e cozimento conveniente de massa preparada com farinhas, amido, féculas fermentadas, ou não, e outras substâncias alimentícias (BRASIL, 1978). De acordo com Pareyt et al. (2009) e Gökmen, Serpen e Açar (2008), os cookies são produtos assados com adição de cereais, altos níveis de açúcar e de gordura e baixos níveis de água (1-5\%).

Este trabalho teve como objetivo produzir e avaliar biscoitos tipo cookies elaborados com substituição parcial da farinha de trigo por farinha de casca de laranja rica em pectina e fibras alimentares, aproveitando o resíduo de forma saudável.

\section{Desenvolvimento}

\section{Matéria Prima}

Os resíduos da laranja foram cedidos por um restaurante da Universidade do Vale do Taquari - Univates após o preparo de sucos, sendo estes higienizados adequadamente antes do procedimento para a obtenção 
da farinha. Os demais ingredientes foram adquiridos em estabelecimentos comerciais da cidade de Lajeado, RS.

\section{Elaboração da farinha da casca de laranja}

Para a preparação da farinha obtida a partir das cascas de laranja, separouse a casca e albedo e higienizou-se em água corrente. Após, as cascas foram colocadas em estufa com circulação de ar a $40^{\circ} \mathrm{C} \pm 2^{\circ} \mathrm{C}$ por aproximadamente 21 horas até a completa secagem. As amostras foram trituradas em liquidificador até a obtenção de uma farinha homogênea e armazenadas em temperatura ambiente até o momento das análises.

\section{Elaboração dos biscoitos}

Para a elaboração dos biscoitos tipo cookies, adaptou-se a metodologia escrita por Rodrigues et al. (2007) e sua preparação foi realizada no laboratório de alimentos da Universidade do Vale do Taquari - Univates, após testes para adequação da formulação final. A farinha de trigo foi substituída por 10, 20 e $30 \%$ de farinha de casca de laranja e os demais ingredientes são: açúcar, ovo, margarina e bicarbonato de sódio.

Os ingredientes foram pesados em balança analítica e misturados, a massa foi homogeneizada manualmente e dividida em pequenas porções em formato circular. Os biscoitos foram assados em formas de alumínio em forno com circulação de ar a $180^{\circ} \mathrm{C}$ por 35 minutos. Após assados, os biscoitos foram resfriados em temperatura ambiente e triturados para a realização das análises.

\section{Análises Físico-químicas}

As análises de atividade de água foram realizadas com o auxílio de um higrômetro (AquaLab - Water Activity Meter, Decagon Devices, Inc. Washington, USA). O teor de umidade foi determinado colocando as amostras de biscoitos em estufa (DeLeo Equipamentos para laboratórios, modelo A3'AFD, Bento Gonçalves, RS, Brasil) a $105^{\circ} \mathrm{C} \pm 5^{\circ} \mathrm{C}$ por 21 horas até a obtenção de peso constante. Para determinação do teor de cinzas, as amostras foram colocadas em mufla (Marconi Equipamentos para Laboratórios, modelo MA $385 / 2$, Piracicaba, SP, Brasil) a $550^{\circ} \mathrm{C} \pm 10^{\circ} \mathrm{C}$ até a obtenção de cinzas brancas ou acinzentadas (INSTITUTO ADOLFO LUTZ, 2008).

A partir da análise do teor de cinza, determinou-se a concentração de sódio, cálcio e potássio dos biscoitos tipo cookies com adição de farinha de casca de laranja com o auxílio de um fotômetro de chama (Digicrom Analytical Ltda., modelo DM-62, São Paulo, SP, Brasil) previamente calibrado. As cinzas foram diluídas, filtradas e avolumadas com a adição de agentes inibidores, óxido de lantânio e cloreto de césio (SKOOG; HOLLER; NIEMAN, 2002). 
O teor de lipídios foi realizado por método gravimétrico, destilação em Soxhlet (Tecnal Equipamentos para Laboratórios Ltda., modelo TE-044-8/30, Piracicaba, SP, Brasil) e foi calculado a partir da expressão

$$
\% \text { Lipídios }(\mathrm{m} / \mathrm{m})=\frac{100 \times \mathrm{N}}{\mathrm{P}} \text {, }
$$

em que $\mathrm{N}=\mathrm{n}^{\circ}$ de gramas de lipídios e $\mathrm{P}=\mathrm{n}^{\mathrm{o}}$ de gramas da amostra.

O teor de proteínas foi realizado através da determinação do teor de nitrogênio total pelo método de Kjeldahl, utilizando bloco digestor (Equipamentos para Laboratórios Ltda, modelo MA 850/16, Piracicaba, SP, Brasil) e destilador de nitrogênio (Tecnal Equipamentos para Laboratórios Ltda., modelo TE-044-8/30, Piracicaba, SP, Brasil). A concentração de proteína das amostras foi calculado através da expressão

$$
\% \text { de proteínas }(\mathrm{m} / \mathrm{m})=\frac{\mathrm{V} \times 0,14 \times \mathrm{f}}{\mathrm{P}}
$$

em que $\mathrm{V}=$ diferença entre o $\mathrm{n}^{\circ}$ de $\mathrm{mL}$ de ácido sulfúrico $0,05 \mathrm{M}$ e o $\mathrm{n}^{\circ}$ de $\mathrm{mL}$ de hidróxido de sódio $0,1 \mathrm{M}$ gastos na titulação, $\mathrm{P}=\mathrm{n}^{\circ}$ de $\mathrm{g}$ da amostra e $\mathrm{f}=$ fator de conversão. $\mathrm{O}$ teor de carboidratos das amostras foi calculado pela diferença dos demais componentes (INSTITUTO ADOLFO LUTZ, 2008).

Todas as análises foram realizadas em triplicatas e os resultados apresentados são as médias das triplicatas.

\section{Análise Sensorial}

Para a realização da análise sensorial foram utilizadas todas as amostras dos biscoitos em pequenas quantidades. A avaliação sensorial foi realizada no laboratório de alimentos da Univates com 50 voluntários não treinados de ambos os sexos. As amostras foram servidas com números aleatórios de três dígitos.

A análise sensorial foi realizada por meio de aceitação, utilizando-se uma escala estruturada em nove pontos os quais variam entre os extremos: 9 gostei muitíssimo e 1 desgostei muitíssimo (DUTCOSKY, 2011). Para analisar a intenção de compra, foi utilizado uma escala variando de: 1 - certamente compraria e 5 - certamente não compraria. (INSTITUTO ADOLFO LUTZ, 2008). As características analisadas foram aparência, odor, sabor, textura e impressão global.

Para o cálculo de Índice de Aceitabilidade (IA) do produto, foi adotada a expressão

$$
\mathrm{IA}(\%)=\mathrm{A} \times 100 / \mathrm{B} \text {, }
$$

em que, $A=$ nota média obtida para o produto e $B=$ nota máxima dada ao produto. O Índice de Aceitabilidade com boa repercussão tem sido considerado $\geq 70 \%$ (DUTCOSKY, 2011). 


\section{Resultados e Discussões}

\section{Análises físico-químicas}

A Tabela 1 apresenta os valores da composição centesimal dos biscoitos tipo cookies elaborados com a substituição parcial da farinha de trigo por farinha de casca de laranja.

Tabela 1 - Valores da composição centesimal dos biscoitos tipo cookies elaborados com a substituição parcial da farinha de trigo por farinha de casca da laranja

\begin{tabular}{l|c|c|c|c}
\hline & Controle & Biscoito 10\% & Biscoito 20\% & Biscoito 30\% \\
\hline Umidade (\%) & $2,75 \pm 0,04^{\mathrm{a}}$ & $2,46 \pm 0,04^{\mathrm{a}}$ & $3,23 \pm 0,09^{\mathrm{a}}$ & $4,05 \pm 0,04^{\mathrm{b}}$ \\
\hline Aw & $0,212 \pm 0,020^{\mathrm{a}}$ & $0,198 \pm 0,060^{\mathrm{a}}$ & $0,279 \pm 0,010^{\mathrm{b}}$ & $0,281 \pm 0,020^{\mathrm{b}}$ \\
\hline Cinza (\%) & $0,82 \pm 0,01^{\mathrm{a}}$ & $1,01 \pm 0,02^{\mathrm{ab}}$ & $1,26 \pm 0,01^{\mathrm{bc}}$ & $1,51 \pm 0,01^{\mathrm{c}}$ \\
\hline Lipídios (\%) & $16,95 \pm 0,04^{\mathrm{a}}$ & $15,72 \pm 0,02^{\mathrm{b}}$ & $13,31 \pm 0,01^{\mathrm{c}}$ & $12,73 \pm 0,02^{\mathrm{c}}$ \\
\hline Proteínas (\%) & $7,89 \pm 0,02^{\mathrm{a}}$ & $7,58 \pm 0,04^{\mathrm{a}}$ & $7,05 \pm 0,05^{\mathrm{a}}$ & $6,62 \pm 0,01^{\mathrm{a}}$ \\
\hline Carboidratos (\%) & $71,59 \pm 0,02^{\mathrm{a}}$ & $73,23 \pm 0,06^{\mathrm{a}}$ & $75,15 \pm 0,04^{\mathrm{a}}$ & $75,09 \pm 0,01^{\mathrm{a}}$ \\
\hline Na (mg/100 g) & $51,99 \pm 4,94^{\mathrm{a}}$ & $56,17 \pm 0,10^{\mathrm{a}}$ & $85,15 \pm 0,20^{\mathrm{b}}$ & $87,64 \pm 0,20^{\mathrm{b}}$ \\
\hline Ca (mg/100 g) & $3,22 \pm 0,63^{\mathrm{a}}$ & $0,94 \pm 0,14^{\mathrm{b}}$ & $5,98 \pm 1,34^{\mathrm{c}}$ & $6,16 \pm 1,20^{\mathrm{c}}$ \\
\hline $\mathrm{K}(\mathrm{mg} / 100 \mathrm{~g})$ & $59,07 \pm 3,54^{\mathrm{a}}$ & $78,88 \pm 0,20^{\mathrm{a}}$ & $110,8 \pm 1,90^{\mathrm{b}}$ & $140,60 \pm 2,00^{\mathrm{c}}$ \\
\hline
\end{tabular}

Média \pm desvio padrão. Significativo pelo teste ANOVA $(\mathrm{p}<0,05)$. Letras iguais na mesma linha não possuem diferença significativa pelo teste de Tukey $(\mathrm{p}>0,05)$;

O teor de umidade das amostras varia de 2,75 a 4,05\% p/p e está de acordo com o padrão estabelecido pela Anvisa (1978) que determina um padrão máximo de $14 \% \mathrm{p} / \mathrm{p}$ de umidade em biscoitos. Pode-se observar que ao aumentar a concentração da farinha de casca de laranja nas formulações dos biscoitos tipo cookies, aumenta o teor umidade dos biscoitos. Esse aumento pode estar relacionado ao fato de que a farinha da casca de laranja possivelmente é um resíduo com elevada retenção de água. Ainda, os biscoitos se enquadram com o apresentado por Sarantópoulos, Oliveira e Canavesi (2011), que estabelece que o teor de umidade em biscoitos entre 2 e $8 \%$ é o que confere crocância ao produto. Estes resultados são menores que os encontrados por Santos, Storck e Fogaça (2014) (11 a 12,5\%) no desenvolvimento de biscoitos com adição de farinha de casca de limão.

Avaliando a atividade de água dos biscoitos tipo cookies, observa-se que estes são um produto estável do ponto de vista microbiológico, sendo que os resultados variaram de 0,198 a 0,281 . Estes resultados são satisfatórios, pois de acordo com Fennema (2000), valores de atividade de água acima de 0,80 e 0,88 propiciam o desenvolvimento de bolores e leveduras. 
O teor de cinza dos biscoitos varia de 0,82 a 1,51\% e estão dentro do padrão estabelecido pela Anvisa (1978), que é de 3\% p/p de resíduo mineral fixo. Observa-se que quanto maior a concentração de farinha de casca de laranja nos biscoitos, maior é a quantidade de resíduo mineral, ou seja, quanto maior a concentração de farinha de casca de laranja maior a quantidade de minerais presente nas amostras. Mas, esses resultados não são essencialmente da mesma composição que a matéria mineral presente originalmente no alimento, pois pode haver a volatilização ou alguma interação entre os compostos da amostra durante a calcinação em mufla (INSTITUTO ADOLFO LUTZ, 2008). Resultados semelhantes foram obtidos por Santos, Storck e Fogaça (2014) que encontraram um teor de cinzas entre 1,29 a 1,53\% nos biscoitos com adição de farinha de casca de limão.

O teor de lipídios das amostras varia de 12,73 e 16,95\%, sendo que quanto maior a concentração de casca de laranja no biscoito tipo cookies, menor a concentração de lipídios. Os resultados obtidos são maiores do que os encontrados por Santos, Storck e Fogaça (2014) (7,47 a 8,27\%) no desenvolvimento de biscoitos com adição de farinha de casca de limão. Essa diferença se deve ao fato de que na formulação dos biscoitos tipo cookies há a adição de margarina e nos biscoitos com adição de farinha de casca de limão não.

Quanto ao teor de proteínas, não houve diferença significativa $(\mathrm{p}<0,05)$ entre as diferentes formulações de biscoitos. $\mathrm{O}$ teor de carboidratos varia de 71,59 até $75,15 \%$ e não houve diferença significativa $(\mathrm{p}<0,05)$ entre as diferentes formulações de biscoitos. Os resultados obtidos foram semelhantes aos encontrados por Santos, Storck e Fogaça (2014) que determinaram um teor de carboidratos entre 69 e 70,1 \% nos biscoitos com adição de farinha de casca de limão.

Analisando os resultados do teor de minerais, percebe-se que quanto maior a quantidade de farinha de casca de laranja no biscoito maior é a concentração dos minerais. $\mathrm{O}$ mineral mais abundante é o potássio que varia de 59,07 a 140,6 mg/100 g, seguido pelo sódio, 51,99 a 87,64 mg/100 g e pelo cálcio que varia de 0,94 a $6,16 \mathrm{mg} / 100 \mathrm{~g}$. Em um estudo da composição mineral de biscoitos elaborados a partir de farinhas de amêndoa ou amendoim adicionadas de ferro, Granato, Piekarski e Ribani (2009) encontraram resultados semelhantes para potássio (182,40 e 169,60 mg/100 g), resultados inferiores para sódio (18,95 e 24,35 mg/100 g) e resultados superiores para cálcio (110,40 e 29,40 mg/100 g), demonstrando a influência de diferentes farinhas na composição nutricional do alimento.

\section{Análise Sensorial}

A Tabela 2 apresenta os resultados da análise sensorial para os biscoitos tipo cookies com substituição parcial da farinha de trigo por farinha da casca de 
laranja. Na análise estatística dos resultados de odor, sabor e impressão global, foi encontrado diferença significativa entre as amostras com as diferentes concentrações da substituição da farinha de trigo por farinha de casca de laranja em comparação com comparando o controle (100\% farinha de trigo), com $\mathrm{p}<0,05$ pelo teste ANOVA. Os resultados de aparência e textura dos biscoitos não foram significativamente diferentes. Esses resultados indicam que o sabor e o odor dos biscoitos tipo cookies são influenciados com o aumento das concentrações de farinha de casca de laranja na composição, mas não interferem na aparência e na textura do biscoito.

Além disso, as notas mostram a aceitabilidade sensorial de sabor e aspectos visuais como aparência e textura. $\mathrm{O}$ índice de aceitabilidade variou de 70,1 a $89,5 \%$ sendo que os biscoitos com o maior índice de aceitabilidade foram os biscoitos com 10 e $20 \%$ de farinha de casca de laranja (IA $=85,3$ e $89,9 \%$, respectivamente) e o biscoito com menor índice de aceitabilidade foi o com $30 \%$ de farinha de casca de laranja (IA $=70,1 \%$ ).

Tabela 2 - Análise sensorial dos biscoitos tipo cookies com substituição parcial da farinha de trigo por farinha da casca de laranja

\begin{tabular}{c|c|c|c|c}
\hline Tratamento & Controle & Biscoito 10\% & Biscoito 20\% & Biscoito 30\% \\
\hline Aparência & $6,28^{\mathrm{a}}$ & $6,68^{\mathrm{a}}$ & $6,94^{\mathrm{a}}$ & $6,05^{\mathrm{a}}$ \\
\hline Odor & $6,20^{\mathrm{a}}$ & $7,17^{\mathrm{b}}$ & $7,20^{\mathrm{b}}$ & $6,51^{\mathrm{a}}$ \\
\hline Sabor & $6,00^{\mathrm{a}}$ & $7,00^{\mathrm{b}}$ & $7,48^{\mathrm{c}}$ & $6,37^{\mathrm{d}}$ \\
\hline Textura & $5,60^{\mathrm{a}}$ & $7,43^{\mathrm{a}}$ & $6,88^{\mathrm{a}}$ & $6,40^{\mathrm{a}}$ \\
\hline Impressão global & $6,25^{\mathrm{a}}$ & $7,05^{\mathrm{a}}$ & $7,14^{\mathrm{b}}$ & $6,54^{\mathrm{c}}$ \\
\hline
\end{tabular}

Significativo pelo teste ANOVA $(\mathrm{p}<0,05)$. Letras iguais na mesma coluna não possuem diferença significativa pelo teste de Tukey $(\mathrm{p}>0,05)$.

A Tabela 3 apresenta os resultados obtidos na análise sensorial das amostras para o teste de intenção de compra, os quais mostram com que frequência as amostras seriam consumidas. Observa-se que as formulações com 10 e $20 \%$ de farinha de casca de laranja aparecem em destaque, sendo que $45,7 \%$ dos avaliadores possivelmente comprariam os biscoitos com essas formulações e $20 \%$ certamente compraria. 
Tabela 3 - Resultados do teste de intenção de compra dos biscoitos tipo cookies com substituição parcial da farinha de trigo por farinha da casca de laranja

\begin{tabular}{c|c|c|c|c}
\hline Tratamento & Controle & Biscoito 10\% & Biscoito 20\% & Biscoito 30\% \\
\hline Certamente compraria (\%) & 11,40 & 20,00 & 20,00 & 22,90 \\
\hline Possivelmente compraria (\%) & 22,90 & 45,70 & 45,70 & 22,90 \\
\hline $\begin{array}{c}\text { Talvez compraria/talvez não } \\
\text { compraria (\%) }\end{array}$ & 22,90 & 20,00 & 22,90 & 17,10 \\
\hline $\begin{array}{c}\text { Possivelmente não compraria (\%) } \\
\text { Certamente não compraria (\%) }\end{array}$ & 20,00 & 14,30 & 11,40 & 22,90 \\
\hline
\end{tabular}

Com esses resultados, observa-se que é possível substituir parcialmente a farinha de trigo por farinha da casca de laranja, aumentando seu valor nutricional sem afetar a aceitabilidade dos biscoitos.

\section{Conclusão}

Os biscoitos tipo cookies com a substituição parcial da farinha de trigo por farinha de casca de laranja apresentaram resultados de acordo com a legislação nacional de alimentos, segundo a composição centesimal, e concentrações elevadas de minerais o que enriquece nutricionalmente o produto. Além disso, a análise sensorial mostra a aceitabilidade do produto, sendo que os biscoitos com 10 e $20 \%$ de farinha de casca de laranja foram os biscoitos com o maior índice de aceitabilidade

Portanto, conclui-se que o uso da casca de laranja na fabricação de farinha para biscoitos pode ser uma alternativa de reduzir e evitar o descarte desse resíduo, além de ser fonte com elevado potencial nutricional.

\section{Referências}

ASSOCIAÇÃO BRASILEIRA DAS INDÚSTRIAS DE BISCOITOS, MASSAS ALIMENTÍCIAS E PÃES \& BOLOS INDUSTRIALIZADOS (ABIMAPI). Estatística - Biscoito. Disponível em: <https://www.abimapi.com.br/estatistica-biscoito.php> Acessado em 6 de dezembro de 2018.

ANUÁRIO DA AGRICULTURA BRASILEIRA [AGRIANUAL]. 2016. Informa Economics FNP, São Paulo, SP, Brasil.

BRASIL. Agência Nacional de Vigilância Sanitária. Gerência-Geral Alimentos Resolução - CNNPA n ${ }^{\circ}$ 12, de 1978, disponível em <http:/ /www.anvisa.gov.br/ anvisalegis/resol/12_78_biscoitos.htm> Acessado em 10 de setembro de 2015. 
BRUNO, M. E. C.; CAMARGO, C. R. O. Enzimas proteolíticas no processamento de biscoitos e pães. Sociedade Brasileira de Ciência e Tecnologia de Alimentos, v. 29, n. 2, p. 170-178, 1995.

CLEMENTE, E.; FLORES, A. C.; ROSA, C. I. L. F.; OLIVEIRA, D.M. Características da Farinha de Resíduos do Processamento de Laranja. Revista Ciências Exatas e Naturais, v.14, n. 2, p. 257-269, 2012.

CHEVALLIER, S.; COLONNA, P.; DELLA VALLE, G.; LOURDIN, D. Contribution of major ingredients during baking of biscuit dough systems. Journal of Cereal Science, v. 31, n. 3, p. 241-252, 2000.

DEITOS, A.; KIELING, A. G.; MORAES, C. A. M.; BREHM, F. A. Avaliação do resíduo casca de laranja na obtenção de pectina e óleo essencial. In: IX Simpósio Internacional de Qualidade Ambiental, 2014.

DUTCOSKY, S. D. Análise Sensorial de Alimentos. 3. ed. Curitiba: Champagnat, 2011. $426 \mathrm{p}$.

FENNEMA, O. R. Química de los alimentos. 2. ed. Zaragoza: Acribia, 2000. 110 p.

FOOD AND AGRICULTURE ORGANIZATION OF THE UNITED NATIONS [FAO]. 2017. Crops. Disponível em: <http:/ / www.fao.org/ faostat/en/\#data/QC>. Acesso em: 6 dez. 2018.

GÖKMEN, V.; SERPEN, A.; AÇAR, O. C.; MORALES, F. J. Significance of furosine as heat-induced marker in cookies. Journal of Cereal Science, v. 48, n. 3, p. 843-847, 2008.

GRANATO, D.; PIEKARSKI, F. V. B. W.; RIBANI, R. H. Composição mineral de biscoitos elaborados a partir de farinhas de amêndoa ou amendoim adicionadas de ferro. Pesquisa Agropecuária Tropical, v. 39, n. 2, p. 92-97, 2009.

GUIMARÃES, R.; BARROS, L.; BARREIRA, J. C.; SOUSA, M. J.; CARVALHO, A. M.; FERREIRA, I. C. Targeting excessive free radicals with peels and juices of citrus fruits: Grapefruit, lemon, lime and orange. Food and Chemical Toxicology, v. 48, n. 1, p. 99106, 2010.

GUTKOSKI, L. C.; NODARI, M. L.; JACOBSEN NETO, R. Avaliação de farinhas de trigos cultivados no Rio Grande do Sul na produção de biscoitos. Ciência e Tecnologia de Alimentos, v. 23, p. 91-97, 2003.

IBGE. Instituto Brasileiro de Geografia e Estatística. 2010. Disponível em http:/ / www.ibge.gov.br/home/estatistica/indicadores/agropecuaria/lspa/lspa_201001_5. sht. Acesso em 10 de setembro de 2015.

INSTITUTO ADOLFO LUTZ. Métodos físico-químicos para análise de alimentos. São Paulo: Instituto Adolfo Lutz, 2008. 1020 p. 
ISHIMOTO, F. Y.; HARADA, A. I.; BRANCO, I. G.; W. A. S. COUTINHO, M. R. Aproveitamento Alternativo da Casca do Maracujá-Amarelo (Passiflora edulis f. Var. flavicarpa Deg.) para Produção de Biscoitos. Revista Ciências Exatas e Naturais, v. 9, n. 2, p. 279-292, 2007.

LI, B. B.; SMITH, B.; HOSSAIN, Md. M. Extraction of phenolics from citrus peels: I. Solvent extraction method. Separation and Purification Technology, v. 48, n. 2, p. 182-188, 2006.

MATOS, E. H. S. F. Dossiê Técnico: Cultivo de limão. Brasília: Centro de Apoio ao Desenvolvimento Tecnológico da Universidade de Brasília - CDT/UnB, 2007.

OLIVEIRA, E. M. S.; RESENDE, E. D. Rendimento da pectina da casca do maracujá em seus estádios diferentes de maturação: verde, maduro e senescência. Ciência e Tecnologia de Alimentos, v. 32, n.3, p. 492-498, 2012.

PAREYT, B.; TALHAOUI, F.; KERCKHOFS, G.; BRIJS, K.; GOESAERT, H.; WEVERS, M. DELCOUR, J. A. The role of sugar and fat in sugar-snap cookies: Structural and textural properties. Journal of Food Engineering, v. 90, n. 3, p. 400-408, 2009.

RODRIGUES, M. A. A.; LOPES, G. S.; FRANÇA, A. S.; MOTTA, S. Desenvolvimento de formulações de biscoitos tipo cookie contendo café. Ciência e Tecnologia de Alimentos, v. 27, n. 1, p. 162-169, 2007.

SANTOS, D. S. D.; STORCK, C. R.; FOGAÇA, A. O. Biscoito com adição de farinha de casca de limão. Ciências da Saúde, Santa Maria, v. 15, n. 1, p. 123-135, 2014.

SARANTÓPOULOS, C. I. G. L.; OLIVEIRA, L. M.; CANAVESI, E. Requisitos de Conservação de Alimentos em Embalagens Flexíveis. Campinas: CETEA/ITAL, 2001. 215 p.

SINDICATO DAS INDÚSTRIAS DE MASSAS E BISCOITOS NO ESTADO DE SÃO PAULO - SIMABESP. A história do biscoito. São Paulo, 2008. Disponível em: <http:/ / www.simabesp.org.br/> Acesso em 15 de setembro de 2015.

SKOOG, D. A.; HOLLER, F. J.; NIEMAN, T. A. Princípios de Análise Instrumental. $5^{\text {a }}$ ed. Porto Alegre: Bookman, 2002. 